\title{
L'instrument 0C0-3 a rejoint la Station spatiale internationale
}

L'instrument OCO-3 est très similaire à OCO-2 et permet de mesurer la colonne de dioxyde de carbone $\left(\mathrm{CO}_{2}\right)$ dans l'atmosphère (figure 1). On rappelle que le premier instrument OCO (Orbiting Carbon Observatory) avait été perdu à la suite d'une défaillance du lanceur. La Nasa avait alors financé la construction d'un instrument et satellite de remplacement $O C O$-2, lancé en 2014 sur une orbite héliosynchrone permettant de mesurer le $\mathrm{CO}_{2}$ en milieu de journée. L'objectif scientifique de cette mission, toujours en fonctionnement, est d'observer les gradients spatiaux et temporels des concentrations pour en déduire les flux de surface (émission et absorption). Les mesures spatiales permettent ainsi de suivre le cycle annuel du $\mathrm{CO}_{2}$ atmosphérique fixé par la photosynthèse (principalement au printemps et en été) et rejeté par la respiration de la végétation et des sols. Des outils dits d' "inversion atmosphérique » servent à estimer les flux de $\mathrm{CO}_{2}$ à partir des mesures, à condition de connaître précisément le transport atmosphérique, déduit des modèles météorologiques (Chevallier et al., 2017). OCO-2 a ainsi permis de rendre compte des anomalies des échanges de carbone entre l'atmosphère, les océans et les terres émergées au cours de l'événement El Niño de 2016.

OCO-3 a été arrimé le 6 mai dernier à la Station spatiale internationale (figure 2), dont l'orbite n'est pas héliosynchrone. Ce n'est pas idéal, car la mesure repose sur le flux solaire réfléchi, qui sera trop faible sur une partie importante des observations. La fraction des observations utiles sera donc plus faible que pour OCO-2, même si certains scientifiques mettent en avant la possibilité pour OCO-3 d'observer le cycle diurne de $\mathrm{CO}_{2}$. Mais OCO-3 est surtout une mission exploratoire pour tester la possibilité de mesurer les émissions des villes et des sites industriels à partir du panache qu'ils génèrent : l'imagerie spatiale permet en effet d'observer les excès de concentration engendrés par les émissions spatialement concentrées. C'est relativement classique pour des gaz tels que le $\mathrm{NO}_{2}$ ou le $\mathrm{CO}$, dont le panache ressort très clairement du fond atmosphérique du fait de leur courte durée de vie. Une mesure similaire pour le $\mathrm{CO}_{2}$ est plus difficile, car le signal

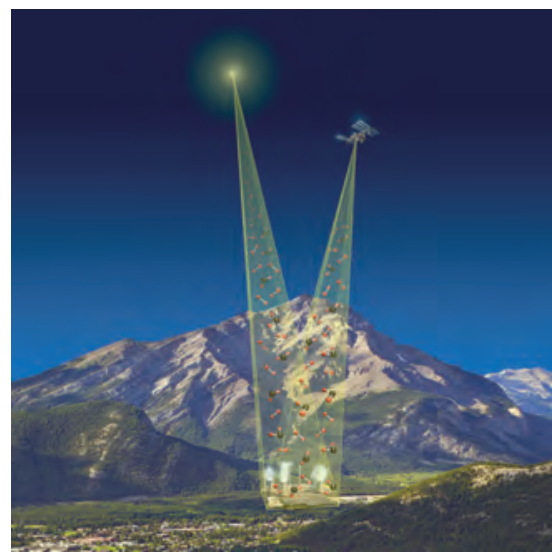

Figure 1. Schéma de principe de la mesure de la colonne atmosphérique de $\mathrm{CO}_{2}$ par 0CO-3. Crédit : Nasa/JPL-Caltech.

généré par un site d'émission tel que la ville de Paris reste petit devant le niveau de fond (typiquement 1 contre 400 ppm) et est comparable au bruit de la mesure. L'extraction du signal du panache nécessite donc des méthodes complexes, encore en développement. L'objectif est bien d'estimer l'émission du site pour valider ou améliorer les inventaires.

Pour permettre une imagerie des villes depuis la station spatiale, l'instrument OCO a été pourvu de miroirs afin de balayer rapidement la zone d'intérêt. L'image de la zone n'est donc pas acquise en une seule fois, mais plutôt en déplaçant le point visé pendant que le satellite défile au-dessus. OCO-3 est ainsi un démonstrateur qui pourrait ouvrir la voie à un suivi depuis l'espace des émissions anthropiques de $\mathrm{CO}_{2}$, avec des missions opérationnelles telles que Sentinel-Carbone, en préparation à l'Agence spatiale européenne. La route est encore longue, car : le niveau de signal est comparable au bruit de mesure ; la précision de l'estimation sera limitée par la précision sur le transport atmosphérique ; la mesure ne pourra donner accès qu'aux émissions des quelques heures précédant le passage du satellite ; l'observation ne peut se faire qu'en ciel clair ; et une part importante des émissions anthropiques ne sont pas « concentrées » et ne pourront pas être mesurées par cette méthode. Ainsi, il est illusoire d'espérer un suivi des émissions anthropiques de $\mathrm{CO}_{2}$ depuis l'espace au cours de la prochaine décennie.

L'instrument MicroCarb, en cours de développement au Cnes et dont le lancement est prévu en 2021, permettra de faire des mesures du même type que celles d'OCO-3, car il est aussi équipé d'un miroir de balayage associé aux capacités de pointage de la plate-forme. Cependant, sa mission principale reste le suivi des flux naturels et l'imagerie des régions fortement émettrices sera exceptionnelle, dans un but exploratoire.

François-Marie Bréon

Laboratoire des sciences du climat et de l'environnement, Institut Pierre-Simon Laplace

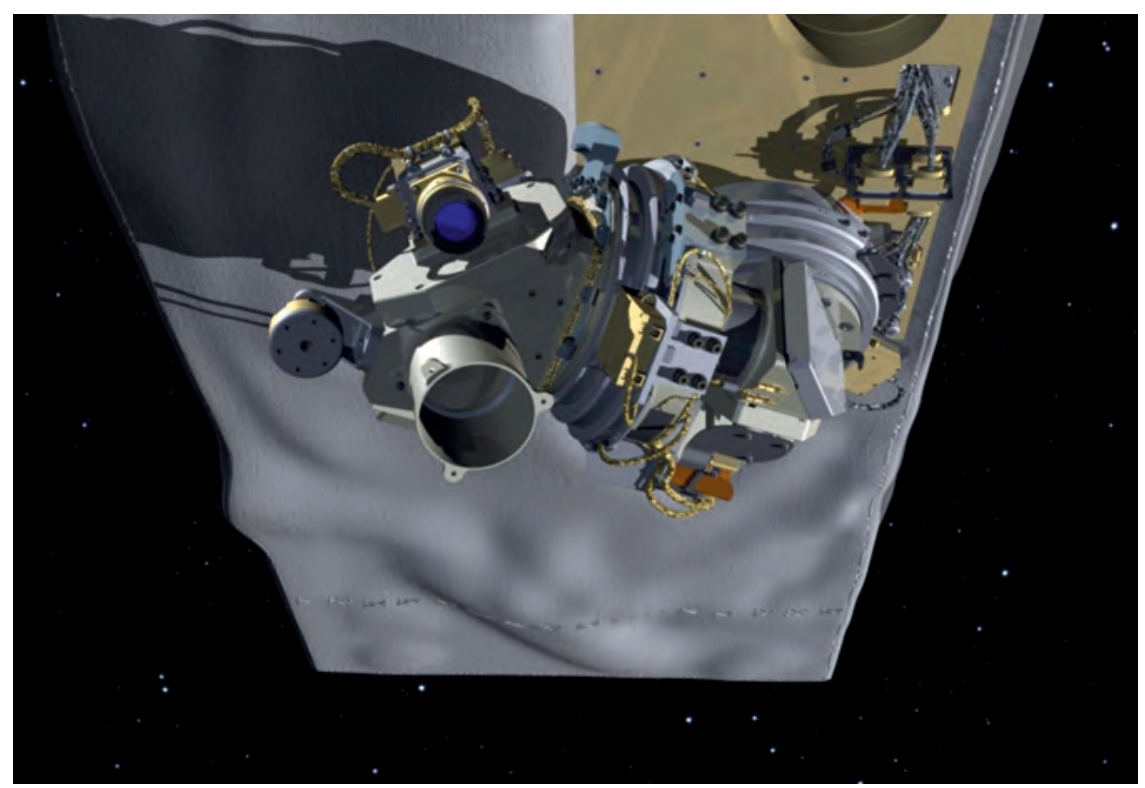

Figure 2. Illustration montrant l'instrument 0C0-3 monté sur la face inférieure de la Station spatiale internationale. Crédit : Nasa/JPL-Caltech. 